\title{
Heterotopic in spontaneous ruptured pregnancy with a living birth term: a case report in Madagascar
}

\author{
Ibrahim Housni*, Romuald Randriamahavonjy, \\ Tanjona Andriamanetsiarivo Ratsiatosika, Rasoanandrianina Bienvenue Solange, \\ Abasse Baco Abdallah, Andrianampanalinarivo Hery Rakotovao
}

Department of Medicine, Antananarivo, Madagascar

Received: 25 April 2018

Accepted: 25 May 2018

*Correspondence:

Dr. Ibrahim Housni,

E-mail: lebibavois087@gmail.com

Copyright: (C) the author(s), publisher and licensee Medip Academy. This is an open-access article distributed under the terms of the Creative Commons Attribution Non-Commercial License, which permits unrestricted non-commercial use, distribution, and reproduction in any medium, provided the original work is properly cited.

\section{ABSTRACT}

Heterotopic pregnancy is defined as the coexistence of an intrauterine pregnancy (UGI) and an ectopic pregnancy (EUS), regardless of its location. It is a rare pathology. Here, we report the case of a 29-year-old patient who had a heterotopic in spontaneous pregnancy complicated by hemoperitoneum at 7 weeks of amenorrhea. A laparotomy with salpingectomy was performed. The result was favourable with a live birth with $37 \mathrm{SA}$ of the GIU. A heterotopic pregnancy must always be eliminated in the presence of acute pelvic pain in the pregnant woman. Laparotomy is a therapeutic alternative if laparoscopy is not available.

Keywords: Acute abdominal pain, Ectopic pregnancy, Heterotopic pregnancy, Salpingectomy

\section{INTRODUCTION}

A heterotopic pregnancy is the simultaneous occurrence of intrauterine pregnancy and ectopic pregnancy. It is a rare pathology; it represents about 1 out of 30000 pregnancies in spontaneous cycle and 1 out of 500 pregnancies in medically procreation..$^{1-3}$

Often a source of misdiagnosis, it is encountered at the stage of complication. So, this constitutes both a diagnostic and therapeutic emergency in cases of rupture ectopic pregnancy. It can be life-threatening with a risk of maternal death of 0.5 out of 100,000 live births. ${ }^{4}$ The challenge for the doctor is to maintain pregnancy in utero and avoid the complications of ectopic pregnancy.

We report a case of heterotopic pregnancy with a history of left oophorectomy, seen at the obstetrics and gynaecology Department of the Soavinandriana Hospital Center with a favourable outcome of pregnancy in utero.

\section{CASE REPORT}

This is about a 35-year-old woman, G3P1, with a history of cesarean section in January 2015 and early spontaneous miscarriage referred for treatment of acute pelvic pain associated with food-induced vomiting. From 5 days to 7 weeks of amenorrhea. The physical examination at the entrance showed a painful abdomen especially at the pelvic level but of low intensity, the plasma b-hCG level was positive and the abdominalpelvic echography revealed a single evolutionary intrauterine pregnancy. In front of this clinical and Para clinical picture, an analgesic treatment was carried out. On the 3rd day of hospitalization, sudden pelvic pain, dagger type, lateralized on the right and examination noted a painful abdomen with defense in the hypo gastric 
region. The abdomino-pelvic echography at emergency showed a hemoperitoneum of moderate abundance, a right lateral-uterine mass and a progressive intrauterine pregnancy. The diagnosis of a heterotopic pregnancy was retained. Facing this clinical and echographic picture, an emergency laparotomy was performed to discover a rupture right ampulla pregnancy with a $300 \mathrm{ml}$ hemoperitoneum associated with clots. The uterus was enlarged and globular [Figure 1]. A right subtotal salpingectomy was performed urgently. The postoperative course was marked by anemia.

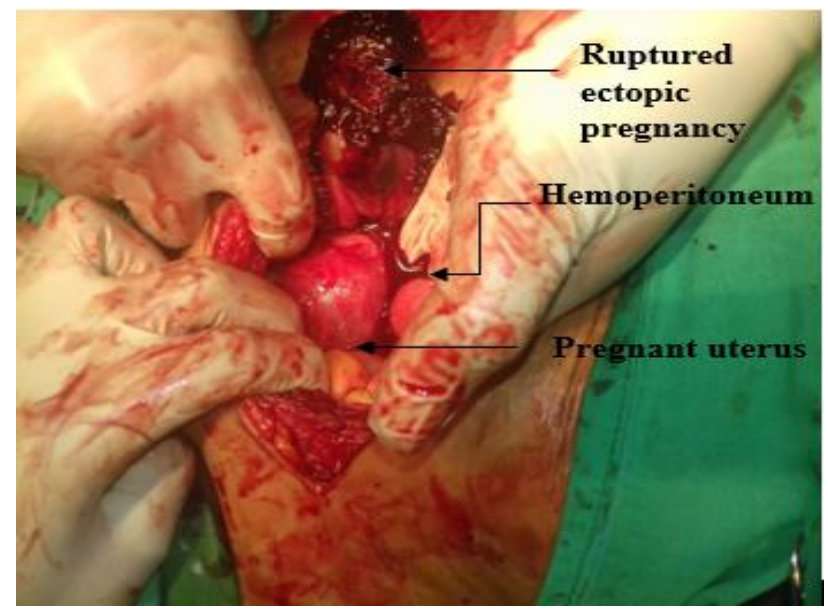

Figure 1: Peroperative view of ruptured ectopic pregnancy.

Intrauterine pregnancy was developing normally and without any particularity. The patient had a loss of clear water at $37 \mathrm{WA}$ and a prophylactic caesarean was performed urgently, to extract a male baby weighing $2700 \mathrm{~g}$ with an Apgar score of 10 in the seventh minute and without any malformation.

\section{DISCUSSION}

Heterotopic pregnancies are rare condition diseases but are becoming more and more frequent as several infertile women use medically assisted procreation., ${ }^{2,5}$ To our knowledge, this is the fourth case reported in Madagascar. Rabarikoto found 2 cases in 2010 at the same Hospital Center and Razafindraibe found 2 cases in 2015 at the Befelatanana University Hospital of Gynecology Obstetric. ${ }^{6}$

The risk factors for heterotopic pregnancy are similar to the risk factors for ectopic pregnancy and medically assisted procreation. ${ }^{1-3,5,7,8}$ In our case, we found a history of pelvic surgery (left oophorectomy and caesarean section) and a history of miscarriage.

The symptomatology may characterize the classic triad of an ectopic pregnancy associated with intrauterine pregnancy. ${ }^{3,9}$ Most often is manifested by isolated lateralized pelvic pain; which corresponds to our case. Diagnosis is difficult if signs of intrauterine pregnancy come first with a threat of abortion or miscarriage. On the other hand, the diagnosis is evoked in front of a table of complications of an ectopic pregnancy like a state of shock or a hemoperitoneum. ${ }^{3,6,9,10}$

For our case, the diagnosis is made late at the stage of ectopic pregnancy ruptured in the presence of a hemoperitoneum. The treatment of ectopic pregnancy is based on surgery (laparoscopy or laparotomy) combined with clinical and echographic monitoring of intrauterine pregnancy. $3,7,9$ For surgery, salpingectomy or salpingotomy may be performed. ${ }^{3,5,9}$

Intrauterine pregnancy usually progresses most often..$^{3,7,9}$ A review of the literature has shown that $66.2 \%$ will give birth alive. ${ }^{11}$ Operative follow-up may be simple or may be marked by complications such as anemia, spontaneous miscarriage or an arrested pregnancy.

\section{CONCLUSION}

To conclude, the diagnosis of a heterotopic pregnancy remains difficult and often late. Facing a pelvic pain, the diagnosis must always be evoked. Although often favorable, the maternal prognosis can be severe by a rupture of the ectopic enlargement. Abdominopelvic and / or transvaginal echography remains a key consideration. Laparoscopic treatment remains the indication of choice; however, laparotomy remains an interesting alternative in case of unavailable technical plateau.

Funding: No funding sources

Conflict of interest: None declared

Ethical approval: Not required

\section{REFERENCES}

1. Kumar R, Dey M. Spontaneous heterotopic pregnancy with tubal rupture and pregnancy progressing to term. Med $\mathbf{J}$ Armed Forces India. 2015 Jul 1;71:S73-5.

2. Kim MJ, Jung YW, Cha JH, Seok HH, Han JE, Seong SJ et al. Successful management of heterotopic cornual pregnancy with laparoscopic cornual resection. Eur J Obstet Gynecol Reprod Biol. 2016 Aug;203:199-203.

3. F. Montilla and colleagues. Heterotopic pregnancy: about a case with a rare clinical picture. j.jgyn. 2006;36:302-5.

4. Creanga AA, Shapiro-Mendoza CK, Bish CL, Zane S, Berg CJ, Callaghan WM. Trends in ectopic pregnancy mortality in the United States: 19802007. Obstet Gynecol .2011;117(4):837-43.

5. Radhouane and colleague. Heterotopic pregnancy: about a case and a review of literature. Imaging of the Woman. 2014;24:177-80.

6. Rabarikoto HF and colleague. 2 cases of spontaneous heterotopic pregnancies at the Soavinandriana Hospital Center, Antananarivo. Review of 
Anesthesia-Resuscitation and Emergency Medicine. 2010;2:21-2.

7. Razafindraibe $\mathrm{AF}$ and colleague. A spontaneous heterotopic pregnancy with a live child born at 34 SA, about a case. JMGO. 2015;2:10-11.

8. Yu Y, Xu W, Xie Z, Huang Q, Li S. Management and outcome of 25 heterotopic pregnancies in Zhejiang, China. Eur J Obstet Gynecol Reprod Biol. 2014 Sep;180:157-61.

9. Torky H, El-Elaa AA. Spontaneous heterotopic triplet pregnancy. Polish Annals of Med. 2017 Aug;24(2):221-3.

10. Mihmanli V, Kilickaya A, Cetinkaya N, Karahisar G, Uctas H. Spontaneous heterotopic pregnancy presenting with hemoperitoneum. J Emerge Med. 2016 Jan;50(1):44-6.

11. Wallach EE, Tal J, Haddad S, Gordon N, TimorTritsch I. Heterotopic pregnancy after ovulation induction and assisted reproductive technologies: a literature review from 1971 to 1993. Fertil Steril. 1996 Jul;66(1):1-2.

Cite this article as: Housni I, Randriamahavonjy R, Ratsiatosika TA, Solange RB, Abdallah AB,

Rakotovao AH. Heterotopic in spontaneous ruptured pregnancy with a living birth term: a case report in Madagascar. Int J Reprod Contracept Obstet Gynecol 2018;7:2942-4. 